\title{
Scalable and Consistent Fabrication of Plasmonic colors via Nanoimprint Lithography
}

\author{
M. Faris Shahin Shahidan ${ }^{\mathrm{a}}$, Jingchao Song ${ }^{\mathrm{a}}$, Timothy D James ${ }^{\mathrm{b}}$, Paul Mulvaney ${ }^{\mathrm{c}}$, and Ann \\ Roberts $^{\mathrm{a}}$ \\ ${ }^{a}$ School of Physics, The University of Melbourne, 3010, Australia \\ ${ }^{\mathrm{b}}$ Reserve Bank of Australia, Craigieburn, Victoria, 3064, Australia \\ ${ }^{\mathrm{c}}$ ARC Centre of Excellence in Exciton Science, School of Chemistry, The University of \\ Melbourne, 3010, Australia
}

\begin{abstract}
We utilised thermal and UV-assisted Nanoimprint Lithography (NIL) i.e. thermal and UV-assisted to produce plasmonic coloration, and compare their ability for scalable fabrication. Several designs are presented and we show the generated colors are dependent on their geometry and the direction of polarisation of incident illumination. Finally, we demonstrated UV-NIL for consistent production of large-area $\left(0.6 \times 0.4 \mathrm{~cm}^{2}\right)$ plasmonic color with extended color gamut.
\end{abstract}

Keywords: Plasmonics, Metasurfaces, Plasmonic colors, Plasmonic coupling, Nanoimprint lithography

\section{INTRODUCTION}

Plasmonic color arises from strong scattering and absorption at characteristic wavelengths due to the excitation of surface plasmons confined to the surface of metallic nanostructures, thus giving rise to non-diffractive coloration. ${ }^{1}$ Unlike dye-based colorant, plasmonic colors are resistant to photobleaching, have low toxicity and enable high-resolution image printing at the diffraction limit while requiring only a reduced set of materials. One widely used plasmonic color configuration incorporates metallic nanoantennas situated above their complementary nanohole screen. Typically, these structures are fabricated using electron beam lithography (EBL). However, recent development in nanoscale embossing techniques such as nanoimprint lithography (NIL) has enable highthroughput and large area nanostructural printing, inaccessible to EBL. As discuss in our previous work, ${ }^{2}$ the 'floating' nanoantenna approach is amenable to upscaling and is compatible with nanoimprint lithography (NIL). Herein, we demonstrate simple fabrication of plasmonic color via thermal and UV-assisted NIL and subsequent metal evaporation. Several geometry designs such as arrays of rods and square nanoantennas have been realized using NIL. Finally, we successfully use UV-NIL to perform a large scale printing of plasmonic color. Our device exhibit either polarisation-dependent or independent coloration under linearly polarised light depending on the specific geometry.

\section{METHODS}

The two main components required to perform NIL are the mould (template) and a resist-coated substrate. First, EBL is used to define the nanostructure pattern on a silicon mould, before treatment with a layer of antisticking coating to reduce adhesion. In thermal NIL, thermopolymer resist is heated above its glass transition temperature $\left(>130^{\circ} \mathrm{C}\right)$ before embossing at high pressure. UV-NIL however, employs a low-viscosity UV curable resist thus requires relatively low embossing pressure at room temperature. Finally, all imprinted samples are evaporated with a layer silver $(\mathrm{Ag})$ film. Arrays of different patterns with varying spatial dimensions were successfully imprinted using both thermal (Figure 1c-f) and UV-NIL (Figure 1h-k). Although capable of highquality pattern replication, thermal NIL involves heating at high temperature which induces defects such as dislocation (Figure 1g) due to the thermal expansion coefficient mismatch between the mould and the substrate. Furthermore, heating and cooling processes consume more fabrication time, reducing throughput.

Further author information: (Send correspondence to M.Shahidan)

M. Shahidan: E-mail: mshahidan@student.unimelb.edu.au 
(a) Thermal Nanoimprint Lithography

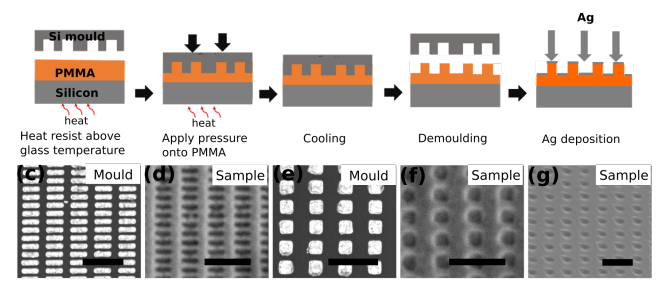

(b) UV Nanoimprint Lithography

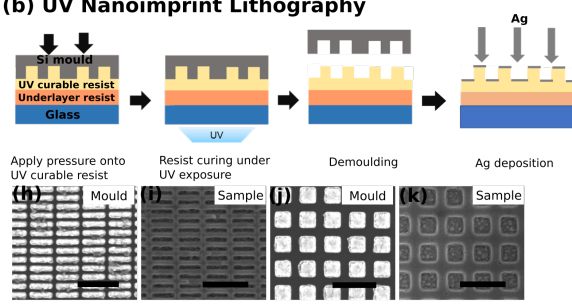

Figure 1. Schematic shows fabrication of plasmonic color via (a) thermal NIL and (b)UV-NIL. SEM images compares the Si mould and the corresponding imprinted sample of different pattern produce from thermal NIL (c-f) and UV-NIL (h-k), while (g) shows defect induced thermally. Scale bar refers to $500 \mathrm{~nm}$

\section{RESULTS}
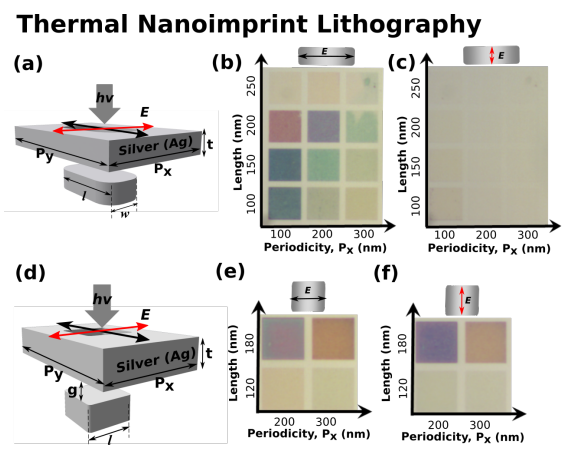
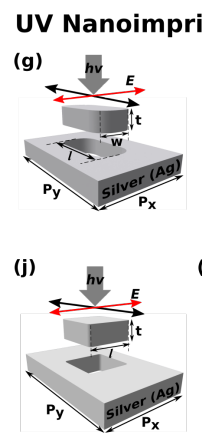
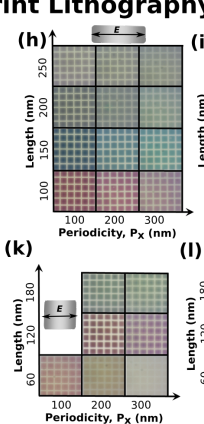
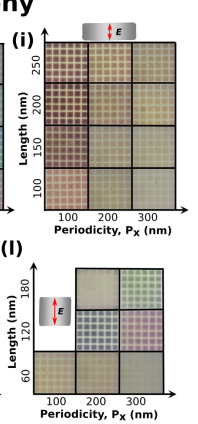
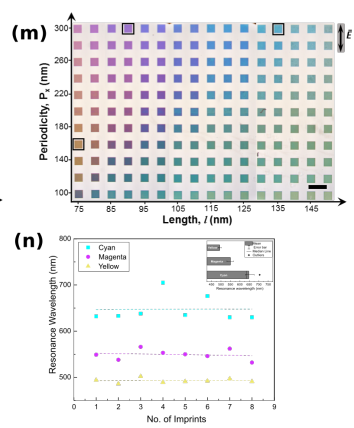

Figure 2. Schematic of nanorod and nanosquare floating antenna produced using thermal NIL, illuminated from film's side (a,d) and using UV-NIL, illuminated from nanoparticle's side (g,j). Optical image showing color generated from nanorods and nanosquare produced using thermal NIL (b,c and e,f) and of UV-NIL (h,i and k,l) under linearly polarised light parallel and perpendicular to long axis of the lattice. $(\mathrm{m})$ Optical image shows color palette generated from the large-area sample produced by UV-NIL. Scale bar refers to $0.4 \mathrm{~mm}$.(n) Plot indicate consistency of color production after several repeated imprint process

The reflected colors shown in Figure 2 are retrieved by illuminating the metallised samples with linearly polarised light at normal incidence, either from the side with the continuous film or with the 'floating' nanorods /nanosquare (Figure 2a,d,g and j). The resulting color varies with length due to the shift in the resonance. The sensitivity of the resulting colors to the direction of polarisation depends on the geometry and duty cycle of the nanostructure. Due to the shortcoming of thermal NIL process mentioned above, we investigated UV-NIL for production of larger scale plasmonic colors $\left(0.6 \times 0.4 \mathrm{~cm}^{2}\right)$ comprising the nanorod structure (Figure $\left.2 \mathrm{~m}\right)$. The plot in Figure 2n indicates consistent color production even after multiple imprints were performed.

\section{ACKNOWLEDGMENTS}

This research was supported by the Australian Research Council through LP160100054. This work was performed in part at Melbourne Center for Nanofabrication (MCN) in the Victorian Node of the Australian National Fabrication Facility (ANFF) and at Swinburne University of Technology

\section{REFERENCES}

[1] Kristensen, A., Yang, J. K., Bozhevolnyi, S. I., Link, S., Nordlander, P., Halas, N. J., and Mortensen, N. A., "Plasmonic colour generation," Nature Reviews Materials 2(1), 16088 (2017).

[2] James, T. D., Mulvaney, P., and Roberts, A., "The plasmonic pixel: large area, wide gamut color reproduction using aluminum nanostructures," Nano letters 16(6), 3817-3823 (2016). 


\section{University Library}

\section{- M M N E R VA A gateway to Melbourne's research publications}

Minerva Access is the Institutional Repository of The University of Melbourne

Author/s:

Shahidan, MFS;Song, J;James, TD;Mulvaney, P;Roberts, A

Title:

Scalable and Consistent Fabrication of Plasmonic colors via Nanoimprint Lithography

Date:

2019-01-01

Citation:

Shahidan, M. F. S., Song, J., James, T. D., Mulvaney, P. \& Roberts, A. (2019). Scalable and Consistent Fabrication of Plasmonic colors via Nanoimprint Lithography. Simpson, MC (Ed.) Juodkazis, S (Ed.) SPIE MICRO + NANO MATERIALS, DEVICES, AND APPLICATIONS 2019, 11201, SPIE-INT SOC OPTICAL ENGINEERING. https://doi.org/10.1117/12.2539079.

Persistent Link:

http://hdl.handle.net/11343/243800 